\title{
Expression of small GTPases in the roots and nodules of Medicago truncatula cv. Jemalong
}

\author{
Abdul Razaque Memon ${ }^{1 \star}$, Christiane Katja Schwager ${ }^{2}$, Karsten Niehaus ${ }^{3}$ \\ ${ }^{1}$ Department of Molecular Biology and Genetics, Faculty of Science and Arts, Usak University, Usak, Turkey \\ ${ }^{2}$ Department of Genome Research, Faculty of Biology, Bielefeld University, Bielefeld, Germany \\ ${ }^{3}$ Department of Proteome and Metabolome Research, Faculty of Biology, Bielefeld University, Bielefeld, Germany
}

\begin{abstract}
In this study we used Medicago truncatula, to identify and analyze the expression of small GTP-binding proteins (Arf1, Arl1, Sar1, Rabs, Rop/Rac) and their interacting partners in the infection process in the roots and nodules. A real-time polymerase chain reaction analysis was carried out and our results showed that Arf1 (AtArfB1c-like), MtSar1, AtRabAle-like, AtRabC1-like, MsRab11-like and AtRop7-like genes were highly expressed in the nodules of rhizobium inoculated plants compared to the non-inoculated ones. On the contrary, AtRabA3 like, AtRab5c and MsRac1-like genes were highly expressed in non-infected nitrogen supplied roots of M. truncatula. Other Rab genes (AtRabA4a, AtRabA4c and AtRabG3a-like genes) were nearly equally expressed in both treatments. Interestingly, $R b o h B$ (a respiratory burst NADPH oxidase homologue) was more highly expressed in rhizobium infected than in non-infected roots and nodules. Our data show a differential expression pattern of small GTP-binding proteins in roots and nodules of the plants. This study demonstrates an important role of small GTP-binding proteins in symbiosome biogenesis and root nodule development in legumes.
\end{abstract}

Keywords: gene expression, Medicago truncatula, root nodules, small GTP-binding proteins

\section{Introduction}

Intracellular membrane trafficking plays a major role in plant growth and development including root hair formation, rhizobium infection process and nodule formation in legumes (Oldroyd and Downie 2008). It is evident from the literature that small GTP-binding proteins perform a critical role in different aspects of root nodule development (Blanco et al. 2009). For example several research groups have shown the importance of Rab1, Rab7 and Rab11F in peribacteroid membrane formation in different legume species like Glycine max, Vigna aconitifolia, Medicago sativa and Medicago truncatula (Cheon et al. 1993, Son et al. 2003, Schiene et al. 2004, Limpens et al. 2009). During rhizobium infection, RabA2 is reported to participate in the polar growth of root hairs of Phaseolus vulgaris (Dalla Via et al. 2017). These authors showed that RabA2 is localized to mobile vesicles around the infection thread (IT) and is required to maintain the integrity of the membrane during IT progression. $\mathrm{Rab} 7$ is reported to be required in vesicular fusion during symbiosome membrane transport in soybean (Cheon et al.
1993). Furthermore, a total of 33 genes of small GTP-binding proteins were identified from a Lotus japonicus genome and among them, some of the Rabs like Rab1, Rab2, Rab5, $R a b 7$, and Rac were predominantly expressed in root nodules of these plants (Borg et al. 1997). Likewise, Schiene et al. (2004) have shown that RabF1 is preponderantly expressed in the nodules and possibly involved in membrane vesicular trafficking and symbiosome formation in M. sativa and $M$. truncatula. Interestingly Rab7 has been shown to be recruited by symbiosome as an endosomal marker when they stop dividing and possibly to regulate the symbiosome maturation process (Clarke et al. 2015). Rab GTPases are the largest member of GTPase family in most of the eukaryotes. They work in combination with Rab effector proteins and regulate nearly all steps of membrane traffic from vesicle budding to vesicle fusion (Pfeffer 2017, Biver et al. 2011). Arf and Sar1 are known to be the key GTP-binding proteins in the regulation of membrane trafficking between ER and GA in plant, yeast and mammalian cells (Pimpl et al. 2000, Ju-

\footnotetext{
*Corresponding author, e-mail: armemon@usak.edu.tr, abdulrezzak.memon@gmail.com
} 
rgens 2004, Memon 2004, Memon 2012). Several observations in A. thaliana and tobacco cells indicate that Arf1 not only functions in retrograde protein transport from Golgi to ER but also in some way affects the anterograde pathway (Memon, 2004). Several isoforms of Arf1 have been identified in the M. truncatula genome (Vernoud et al 2003, Yuksel and Memon 2008) but their exact function in root and/or nodules has not been defined. It will be interesting to examine the possible involvement of these proteins in intracellular vesicle trafficking in the early steps of symbiotic interactions.

Accumulating evidence shows that Rop/Rac acts as a predominant switch in signal transduction in plants and is reported to regulate several developmental processes in plants, such as polarized cell growth, cell morphogenesis, hormone signalling, defence and responses to oxygen deprivation (Craddock et al. 2012). In root hairs (Foreman et al. 2003, Ke et al. 2016, Lei et al. 2015) and in pollen tubes (Potocky et al. 2007), these GTP-binding proteins activate NADPH oxidase (Wong et al. 2007) and are responsible for the apical accumulation of reactive oxygen species (ROS). ROS is shown to be essential for cell elongation and to play a vital role in root hair deformation and curling during rhizobial infection (Damiani et al. 2016a, Damiani et al. 2016b). Recently it has been shown that Rop activates the NADPH oxidase homologue of plants termed Rboh (for respiratory burst oxidase) and generates ROS during early microbial infection (Kiirika et al. 2012).

Membrane protein targeting in the symbiosome varies depending on the mosaic nature of the symbiosome which includes the properties of both plasmalemma and tonoplast. This indicates that the participation of several small GTPbinding proteins in symbiosome membrane biogenesis is more complex and this process could be studied in detail. To elucidate the mechanism of membrane transport in root nodules, we made an attempt to retrieve the EST databases of two model legume plants, Medicago and Lotus and searched for the nodule-specific expressed transcripts of small GTPases (Yuksel and Memon 2008). This analysis showed a group of 10 small GTPases which are likely to be expressed in root nodules and among them, seven belonged to the Rab cladistic group and two belonged to the Arf subfamily group (Yuksel and Memon 2008).

In this study, we made a detailed expression analysis of these GTP-binding proteins in rhizobium inoculated and non-inoculated roots and nodules of $M$. truncatula with the intention of exploring the role of these proteins in rhizobium infection process and symbiosome formation in $M$. truncatula roots.

\section{Materials and methods}

\section{Plant cultivation}

Medicago truncatula cv. Jemalong seeds were surface sterilized in concentrated $\mathrm{HCl}$ (37\%) for $10 \mathrm{~min}$. After several washings with sterile water, seeds were spread on agar plates with plant minimal medium adjusted to $\mathrm{pH} 5.7$ (Rolfe et al.
1980) and germinated for two days in the dark at room temperature. For nodulation, each seedling was inoculated on agar plates with $0.1 \mathrm{~mL}$ Sinorhizobium meliloti 2011 grown to an optical density of 0.6 in YEP medium $\left(5 \mathrm{~g} \mathrm{~L}^{-1}\right.$ yeast extract, $10 \mathrm{~g} \mathrm{~L}^{-1}$ peptone, $5 \mathrm{~g} \mathrm{~L}^{-1} \mathrm{NaCl}, \mathrm{pH}$ 7.0) supplemented with $600 \mathrm{mg} \mathrm{L}^{-1}$ streptomycin. Mature roots and nodules were harvested four weeks after infection. Plants were grown under the following conditions: $25^{\circ} \mathrm{C}, 65 \%$ humidity, 200 $\mu \mathrm{E} \mathrm{m}^{-2} \mathrm{~s}^{-1}$ and $16 \mathrm{~h}$ light/ $8 \mathrm{~h}$ dark. Growth conditions for inoculated and non-inoculated plants were the same except that non-inoculated plants were supplied with $\mathrm{NH}_{4} \mathrm{NO}_{3}$ as the nitrogen source. Nodules and roots were separated and were used for RNA isolation, cDNA synthesis, and quantitative real-time PCR (qPCR).

\section{Real-time qPCR}

Total RNA was isolated from the roots and nodules of $M$. truncatula grown with and without rhizobium with the use of the RNeasy Plant Mini Kit (Qiagen, Hilden, Germany) following the manufacturer's instructions. RNA was quantified using a Nanodrop Analyser 1000 (Thermo Scientific, Wilmington, DE, USA) and cDNA was synthesized from 2 $\mu \mathrm{g}$ of extracted RNA as previously described by (Mehrtens et al. 2005).

Forward and reverse primers of the targeted genes were designed using Primer 3 software (Rozen and Skaletsky 2000) and are listed in Table 1. Real-time qPCR (RT-qPCR) was performed using the Platinum SYBR Green qPCR SuperMix UDG Kit (Invitrogen, Karlsruhe, Germany) following the manufacturer's instructions on a Rotor-Gene 6000 (Corbett Research; http://www.corbettlifescience.com/) cycler. In our preliminary experiments ubiquitin, actin and ribosomal $18 \mathrm{~S}$ genes were used for internal control. Our data showed that ubiquitin (TC102473) was the most suitable for use as an internal control for RT-qPCR under our experimental conditions. Therefore, in our further experiments we used ubiquitin as an internal control in data evaluation.

Raw data were analyzed with the CAmpER software tool implementing an algorithm based on a four parameter logistic model (FPLM) (Tichopad et al. 2003) of the fluorescence curve. The FPLM algorithm was used to calculate efficiency-corrected crossing points (cCPs) that were subsequently analyzed using the REST (Relative Expression Software Tool) software (Tichopad et al. 2003, Bucciarelli et al. 2006). Each experiment was replicated 3 times and each sample was replicated 3 times for real-time qPCR analysis.

\section{Statistical analysis}

All the analyses were performed in triplicate and the results were recorded as mean \pm standard deviation (SD). Visualizations were created using GraphPad Prism 7.5 for windows (GraphPad Software, San Diego USA). Analysis of variance (ANOVA) and post-hoc test (Tukey) was carried out by using IBM SPSS statistics 23 Software. Tukey test was used to evaluate the significance of different treatment groups. 
Tab. 1. Primer pairs used for quantitative real-time PCR. Primer3 software was used to design the primers of the each gene.

\begin{tabular}{|c|c|c|}
\hline Gene & $\begin{array}{l}\text { Forward Primer } \\
\text { Sequence }\left(5^{\prime}-3^{\prime}\right)\end{array}$ & $\begin{array}{l}\text { Reverse Primer } \\
\text { Sequence }\left(5^{\prime}-3^{\prime}\right)\end{array}$ \\
\hline AtRabAle & CGAATCTTCAGTTGAAACCGCTC & GCTGGTCAAGAAAGGTACCGAG \\
\hline$A t R a b A 3$ & ACССТССАСАТТСАСАССAG & GAGGTGCATTAGGAGCAATGC \\
\hline$A t R a b A 4 \mathrm{a}$ & GGCCAAGAACGATATAGAGCAG & TGCATCCTCTGTGGGTACTG \\
\hline AtRabA4c & GTGCGTATTACCGTGGAGCTG & GAGCATCTTCAGTAGGTACTGCC \\
\hline AtRabA5c & GCTGGACAAGAAAGGTTTAGAGC & CCCACCAACATTCTCGCAAC \\
\hline AtRabC1 & GTTCAGGGCAGCAAGAATTCGA & TCCCAAATGGCAAGCTTAAGC \\
\hline AtRAbG3a & CACTTGATAACTGGCACGAGGAG & GGAATGCAGCATCAACATTGAGG \\
\hline MSRab11 & GTGCTGATTGGTGATTCTGG & CTTGATCGCCTCGTTATGTC \\
\hline AtARFB1c & CATCGCTCCTCTCAGGTCTTG & GAGGACACTCTGGAGGCAC \\
\hline AtArlA1d & GAGGACATGATTCCTACAGTGGG & CACTCAATGATGGCTTGCTCAG \\
\hline MtSar1 & CGCTCAGGTGGATGCTGTAGTC & CATGAACACCTCCAAAGGACG \\
\hline MtArf1 & CTGGATGTAGTGGCGCTGT & CCAAGACAAGATTCGTCCACTG \\
\hline$M t-z-C O P-2$ & CTCCTTCCAAACTCGAGTTG & GCTCCAGATTCTGTACTATTAC \\
\hline MSRac1 & GCTTGACCTTCGGGATGATAAG & GACAACTCTTATGGCTGCGTC \\
\hline MtRbohB & GCTCGCTCTGCTCTTATTGC & TGCGCTTGTAGACACTACGC \\
\hline AthRop7 & СTCTTCACCCTGGGCAGTAG & GGCCATTGAGCTATAGAGGAGC \\
\hline Ubiquitin & GCAGATAGACACGCTGGGA & AACTCTTGGGCAGGCAATAA \\
\hline
\end{tabular}

\section{Results}

To obtain a global view of the gene expression of small GTP-binding proteins in roots and nodules of M. truncatula, RT-qPCR experiments were carried out with plants inoculated with S. meliloti for 4 weeks. The efficiency of cDNA synthesized was assessed by endpoint RT-qPCR using constitutive gene-specific primers encoding ubiquitin as a reference gene. Expression patterns of MtRab, MtArf, MtArl, MtSar, MtRop7, $M t R a c 1, M t R b o h B$ and MtCOP in nodules and rhizobia inoculated and non-inoculated roots of $M$. truncatula are shown in Figs. 1, 2, and 3.

The RT-qPCR experiments with these genes showed that AtRabAle and AtRabC1 homologues and MSRab11 were highly expressed in the nodules compared to the roots of non-inoculated plants (see Figs. $\mathrm{la}, \mathrm{f}, \mathrm{h})$. On the other hand, the expression pattern of homologues of AtRabA3, AtRabA4a, AtRabA5c and AtRabG3a was significantly higher in the roots of noninoculated plants compared to the nodules and roots of inoculated plants (see Figs. 1b, c, e, g). In contrast, homologues of $A t R a b A 4 c$ was predominantly expressed both in the roots of inoculated and non-inoculated plants (Fig. 1d).

Inoculation of M. truncatula roots with S. melilo$t i$ caused a strong induction of the MtSar1 transcript (see Fig. 2c). We found that MtSarl expression was induced around 6 times more in inoculated than in the roots of non-inoculated nodules (Fig. 2). Post-hoc test showed that MtSar1 expression in nodules was statistically highly significant $(\mathrm{P}<0.05)$ as compared to the expression in control roots and roots treated with $S$. meliloti. Interestingly ArfB $1 c$ transcript accumulation was significantly reduced in the roots of non-inoculat-
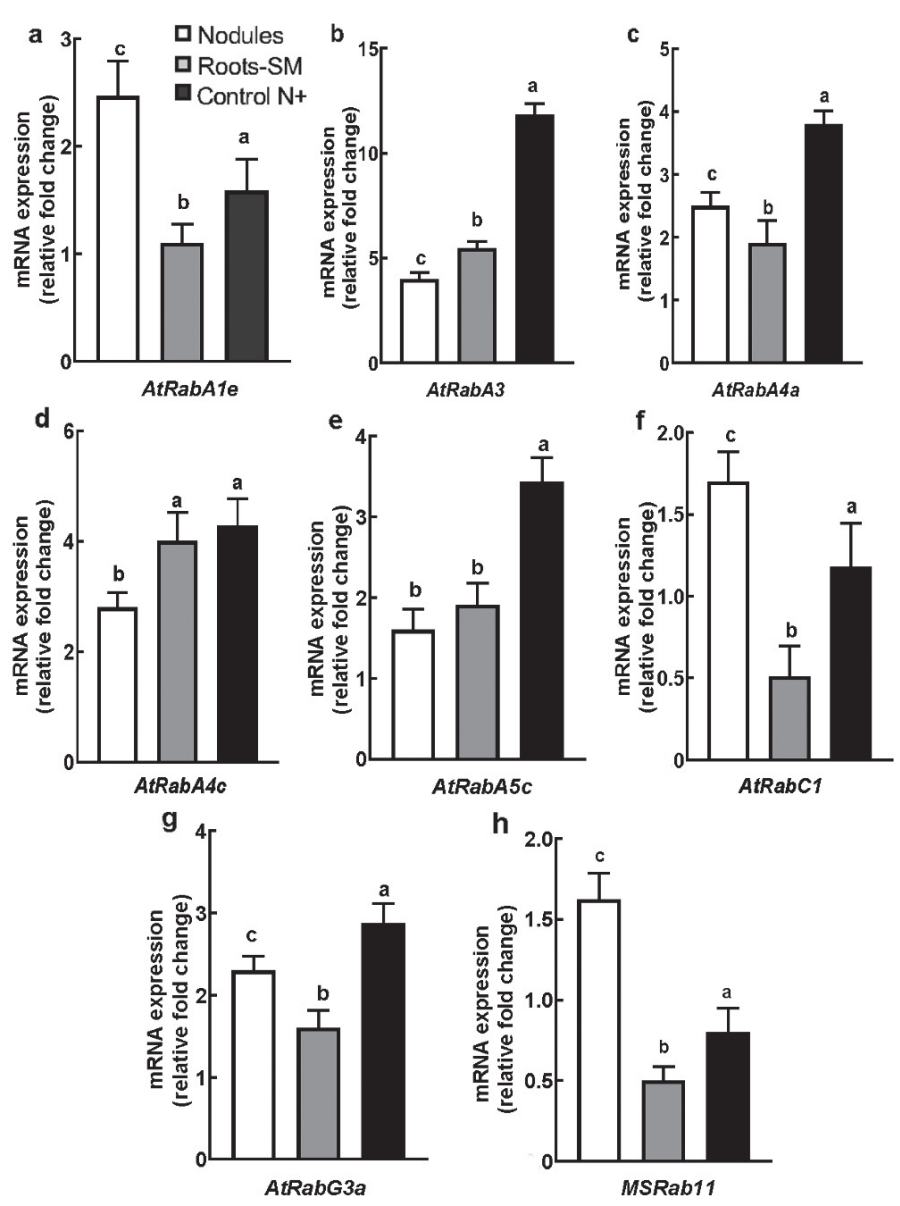

Fig. 1. Expression levels of Rab group GTP binding proteins (at mRNA level) in the nodules and roots (roots-SM) of Medicago truncatula inoculated with rhizobium Sinorhizobium meliloti. Ubiquitin was used as internal control. Control roots (control $\mathrm{N}+$ ) were harvested from un-inoculated plants which were supplied with nitrogen fertilizer. Bars represent standard deviation of three replicates. Different letters show significant differences between groups $(\mathrm{P}<0.05)$ based on Tukey test. 


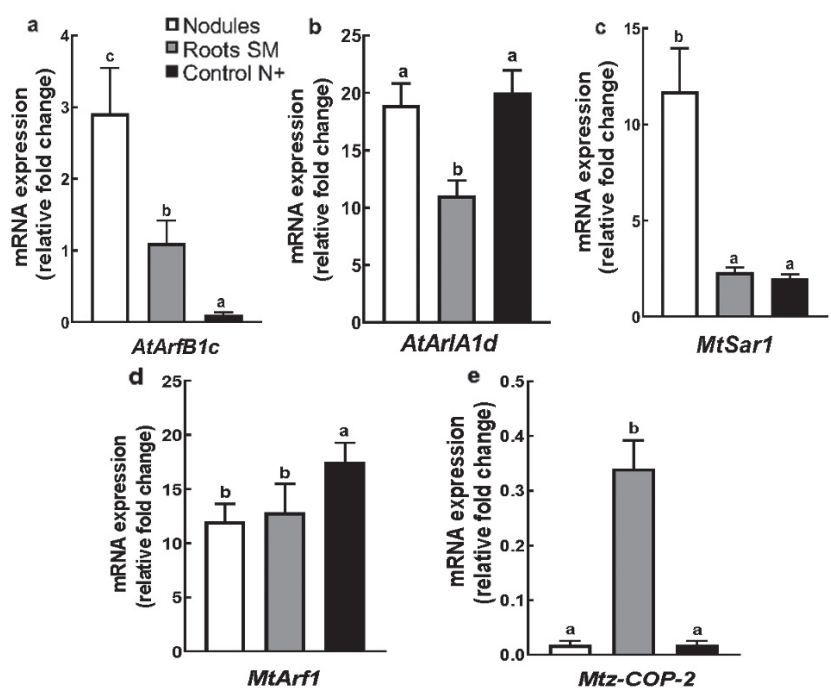

Fig. 2. Expression levels of Arf/Sar group GTP- binding proteins (at mRNA level) in nodules and roots (roots SM) of Medicago truncatula inoculated with rhizobium Sinorhizobium meliloti. Ubiquitin was used as internal control. Control roots (control $\mathrm{N}+$ ) were harvested from un-inoculated plants which were supplied with nitrogen fertilizer. Bars represent standard deviation of three replicates. Different letters show significant differences between groups $(\mathrm{P}<$ 0.05 ) based on Tukey test.

ed plants (Fig. 2a). This clearly suggests that ArfB1c is possibly nodule specific and expressed in host plant roots after rhizobium infection, indicating its importance in nodule development. Intriguingly, another MtArf1(MtrGB57486383) transcript was equally expressed in both roots treated with S. meliloti and nodules of inoculated plants (Fig. 2d) but its expression was increased in non-inoculated roots suggesting its general role in vesicular trafficking.

Our data show that one of the interacting partners of Arf1, a z-subunit of MtCOP2 (a component of COP1 vesicles) was predominantly expressed in rhizobia inoculated roots (Fig. 2e) and this expression pattern was quite different than that of Arf1 expression in roots and nodules. Fig. $2 \mathrm{~b}$ shows that AtArlA1d was highly expressed in both nod- ules and roots of non-inoculated plants and this expression pattern of At ArlA1d was different than that of the expression pattern of the other genes in the ARF/Sar1 group (see Figs. 2 a, b, c, d).

To assess the role of MtRop in the infection process, $M t R o p$ gene expression, and their relative transcriptional levels were analyzed in nodules and roots of inoculated and non-inoculated plants. The RT-qPCR experiments showed that indeed our previously identified transcript (homologues to A. thaliana Rop7 and A8IK57_MedtrRop 8) is predominantly expressed in root nodules (Fig. 3c), indicating its role in nodule biogenesis.

There are few data available related to the participation of NADPH oxidases in Rhizobium and symbiotic interactions in legumes. This prompted us to investigate the Rac and RBOHS role during the initial stages of nodulation (Figs. 3a, b). Our results showed that the expression of $M t R b o h B$ transcript was significantly increased in roots and nodules of inoculated as compared with non-inoculated plants (see Fig. 3b).

\section{Discussion}

The cDNA array analysis performed with $M$. truncatula has identified the genes involved in nitrogen and carbon metabolism (Tesfaye et al. 2006). However, very little is known about the genes and proteins involved in vesicular trafficking which is reported to increase in root cells during the infection process (Navazio et al. 2007, Yuksel and Memon 2008). The symbiotic interaction between rhizobia and roots of legumes results in the formation of an infection thread followed by the formation of a bacteria containing compartment, the symbiosome, which continuously fuses with numerous vesicles and finally gives rise to plant cells colonized by a huge number of bacteroids within mature nitrogen-fixing nodules (Wang et al. 2010, Ivanov et al. 2010). Since a huge amount of membrane material is needed to be transported from ER to the GA for symbiosome formation (Oldroyd and Downie 2008), it is assumed that the transport of the symbiotic membranes should be dramatically enhanced due to rhizobium infection. Consequently, we carried out a differential expression analysis, which provided a comprehensive view of the rate of
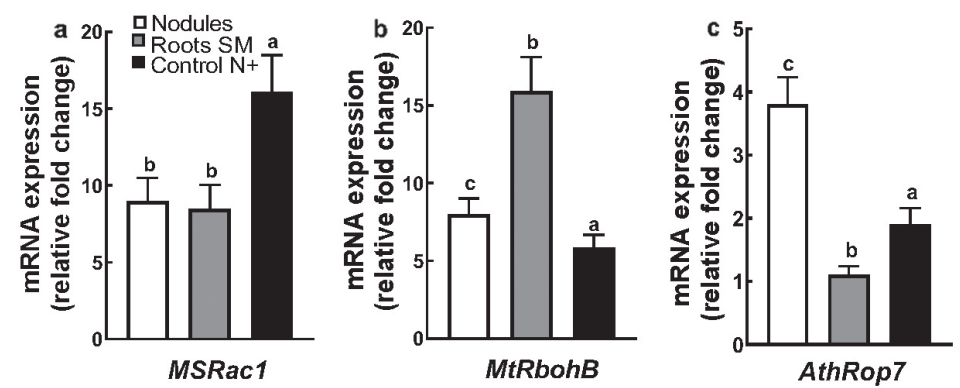

Fig. 3. Expression level of Rop/Rac group GTP binding proteins and Mt Rboh gene in nodules and roots (roots SM) of Medicago truncatula inoculated with rhizobium Sinorhizobium meliloti. Ubiquitin was used as internal control. Control roots (control N+) were harvested from un-inoculated plants which were supplied with nitrogen fertilizer. Bars represent standard deviation of three replicates. Different letters show significant differences between groups $(\mathrm{P}<0.05)$ based on Tukey test. 
transcription of small GTP-binding proteins during nodule development in M. truncatula.

Our previous research conducted on databases of two model legume plants (Medicago and Lotus) containing ESTs of genes expressed in legume-rhizobium infection process have enabled the identification of 14-15 genes of GTP-binding proteins possibly involved in root hair formation and nodule development in M. truncatula (Yuksel and Memon 2008). Analyses of sequence similarities and of the presence of specific family and subfamily conserved motifs in their primary sequence allowed the identification of closest homologues from Arabidopsis and the assignment of around 8 Medicago and lotus sequences to the Rab family, 4 to Arf/Arl/Sar family and 2 to Rop/Rac family involved in root hair formation and nodule development (Yuksel and Memon 2008). Rab proteins have been functionally linked to the development of nodules in legumes (Limpens et al. 2009). For example, Rab1 and Rab7 proteins are known to be part of symbiosome membrane biogenesis (Cheon et al. 1993). Within the Medicago Rab family, the most abundant subclass is the RabA subclade and this is consistent with the significant expansion of the RabA group reported in Arabidopsis (Woollard and Moore 2008). Two Rab gene homologues to AtRabAle and AtRabC1 and one gene homologue to MsRab11 were shown to be dominantly expressed in nodules of rhizobium inoculated plants (Figs. 1a, f, h). Although other Rabs (RabA3, RabA4a, RabA4c, RabA5c RabG3a) were also expressed in nodules and roots of inoculated plants their expression level was not significantly increased compared to the roots of un-inoculated plants (Figs. 1 b,c,d,e,g). The precise role of these Rabs at different stages of infection process cannot be clarified at this stage; however, our gene expression data clearly indicates that these proteins play an important role in symbiosome formation. In Arabidopsis most of the genes in the RabA4 group were spatially expressed in root tissues except AtRabA4d which was specifically expressed in stamen and pollen (Yuksel and Memon 2008, Szumlanski and Nielsen 2009). A subclade of Rab GTPase is reported to be involved in Golgi to plasma membrane trafficking in both plant and mammalian cells (Woolward and Moore 2008), therefore it could fulfil the complementary role in different steps of the exocytic pathway in root cells. Evidence from different sources supports the involvement of RabA GTPases in post Golgi trafficking (Lycett 2008) and could play a substantial role in nodule development. Another RabG3 class ( $R a b G 3 a$ ) is related to $R a b 7$ (Mazel et al. 2004) and has been highly expressed in roots and nodules. This Rab GTPase has been reported an endosomal marker and acquired by symbiosomes when they stop dividing and that Rab7 regulates the maturation of the symbiosomes into nitrogen fixing organelles in M. truncatula (Son et al. 2003, Limpens et al. 2009).

The level of $A r f B 1 c$ expression in the nodules of inoculated plants was significantly higher than in the roots of noninoculated ones. Indeed, very little $A r f B 1 c$ transcript accumulation has been observed in the roots of non-inoculated plants (Fig. 2a). This indicates that in Medicago truncatula, this Arfis possibly a nodule specific and expressed in the host plant roots after rhizobium infection. Intriguingly another MtArf (MtrGB57486383) transcript was expressed both in roots and nodules of inoculated and non- inoculated plants suggesting its general role in vesicular trafficking in plant cells (Fig 2d). In our previous experiment with Arf1 protein expression, we observed a significant increase in Arf1 protein expression with rhizobial infection and the major pool of the Arf1 in both nodules and roots is possibly present in active form (Memon 2012). It is interesting to note that Sar1, which is required in vesicular trafficking in early anterograde trafficking from ER to cis-Golgi, has shown a very high expression in nodules as compared to roots in rhizobium inoculated plants (Fig. 2c). These observations indicate that the genes of these proteins involved in early secretory pathway may play a central role in nodule development and biogenesis (Memon 2012, Oldroyd and Downie 2008). The expression of AtArl A1d was more or less similar in both nodules and non-inoculated roots (Fig. 2b). This suggests the dual role of this $A r l$ in both the early and late secretory pathway. Interestingly the expression pattern of $M t z C O P-2$ was shown to be different than that of AtArfB1c (Figs. 2a, e). These differences in expression could be due to the fact that Arflis not only required in the retrograde pathway from GA to ER transport but also involved in late secretory transport from trans-Golgi to endosomes and to the plasma membrane (Memon 2004, Matheson et al. 2007, Park and Jurgens 2012).

Rop GTP-binding proteins work as signalling molecules to control the polar growth of root hairs, and the formation of the interdigitated pattern and leaf pavement and pollen tube growth in Arabidopsis (Fu et al. 2005, Gu et al. 2005). Recent data show that the spatiotemporal dynamics of Rop proteins, the cytoskeleton, endocytosis, and exocytosis are intertwined (Yalovsky et al. 2008). The expression of some Rops in roots of $M$. truncatula is altered in response to rhizobia infection (Liu et al. 2010). Previously we identified a single Rop gene LjaTC 11919 from Lotus which was predominantly expressed in nodules (Yuksel and Memon 2008) and is closely associated with a gene from $M$. truncatula (Mtr IMGACC140545-16.1). This gene is homologous to AtRop7 and MtrRop8. Our realtime qPCR results showed about 3 times more expression of AthRop7 transcript in the nodules of inoculated plants than in those of non-inoculated one (Fig. 3c), indicating its role in nodule biogenesis. Some reports have shown the increased expression of MtRop5 and MtRop6 after rhizobium inoculation (Riely et al. 2011). It is assumed that these genes could be involved in cytoskeleton reorganization of the host cell during rhizobium infection process.

There is increasing evidence that Rop/Rac proteins are involved in the generation of ROS during the early stages of microbial infection via the activation of respiratory burst NADPH oxidase homologues of plants (RBOH) $(\mathrm{Ke}$ et al. 2016). The ROS are reported to be involved in signalling in response to rhizobia-legume interaction (Pauly et al. 2006, Jamet et al. 2007) and it appears that ROS production is essential for optimal symbiosis formation. Rac and $\mathrm{RBOH}$ seem to play an important role in ROS production 
during symbiotic interaction (Marino et al. 2011, Montiel et al. 2012, Arthikala et al. 2014). Interestingly the downregulation of RbohB through RNAi in Phaseolus vulgaris roots reduced ROS production and lateral root density and greatly impaired nodulation (Montiel et al. 2012). On the contrary, over-expression of RbohB in P. vulgaris significantly enhanced the ROS production, infection thread formation and increased the density of symbiosomes in nodules, and the density and size of bacteroids in symbiosomes (Arthikala et al. 2014). Our data in Fig. 3b showed significantly high expression of $M t R b o h B$ in rhizobium inoculated roots compared to non-inoculated ones. These observations illustrate the importance of the NADPH oxidases and their interacting GTPases (Rops) in Rhizobium infection process and show that is possibly plays an important role in the early infection process. Racl is an orthologue to small GTPase MtROP9 in M. truncatula (Kiirika et al. 2012) and our data show that it was expressed in both roots and nodules but its expression was significantly higher in the roots of non-inoculated plants (Fig 3a). This implies that Racl is not only involved in ROS-mediated early infection process as reported earlier (Kiirika et al 2012) but also involved in the activation of plant defence response. The early defence response of Rac/Rop-like GTPases in plants has been previously reported (Wong et al. 2007). For example Racl in rice activates Rboh mediated ROS signalling and is reported to be a positive regulator in disease resistance.

This work provides important experimental evidence for the involvement of small GTP-binding proteins and the respiratory burst oxidase homologue $(\mathrm{RBOH})$ in Rhizobium infection process and nodule development in the roots of M. truncatula.

\section{References}

Arthikala, M.-K., Sánchez-López, R., Nava, N., Santana, O., Cárdenas, L., Quinto, C. 2014: RbohB, a Phaseolus vulgaris NADPH oxidase gene, enhances symbiosome number, bacteroid size, and nitrogen fixation in nodules and impairs mycorrhizal colonization. New Phytologist 202, 886-900.

Biver, S., Portetelle, D., Vandenbol, M. 2011: Multicopy suppression screen in Saccharomyces cerevisiae strain lacking the Rab GTPase-activating protein Msb3p. Biotechnology Letters 33, 123-129.

Blanco, F. A., Meschini, E. P., Zanetti, M. E., Aguilar, O. M. 2009: A small GTPase of the Rab family is required for root hair formation and preinfection stages of the common bean Rhizobium symbiotic association. Plant Cell 21, 2797-810.

Borg, S., Brandstrup, B., Jensen, T. J., Poulsen, C. 1997: Identification of new protein species among 33 different small GTPbinding proteins encoded by cDNAs from Lotus japonicus, and expression of corresponding mRNAs in developing root nodules. The Plant Journal 11, 237-250.

Bucciarelli, B., Hanan, J., Palmquist, D., Vance, C. P. 2006: A standardized method for analysis of Medicago truncatula phenotypic development. Plant Physiology 142, 207-219.

Cheon, C. I., Lee, N. G., Siddique, A. B., Bal, A. K., Verma, D. P. 1993: Roles of plant homologs of Rablp and Rab7p in the biogenesis of the peribacteroid membrane, a subcellular compartment formed denovo during root nodule symbiosis. European Molecular Biology Organization Journal 12, 4125-35.

\section{Conclusion}

In this communication, we described the role of small GTP-binding proteins (Arf1, Arl1, Sar1, Rabs, Rop/Rac) and some of their interacting components in the biogenesis of the symbiosome membranes in the root nodules of $M$. truncatula. Our gene expression data showed that several Rabs, Rop, Arf and Sar proteins are involved in early and late secretory pathways during rhizobium-legume interaction and nodule development. This study gives an important clue that in addition to the proteins involved in the late secretory pathway (like Rab7, Rab11), the GTP-binding proteins of the early secretory pathway (Sar1, ArfB1c RabA1e, RabC1) are also more highly expressed in rhizobium inoculated roots and nodules than in non-inoculated roots. This suggests that the activation of multiple GTP-binding proteins (both in early and late secretory pathway) is required for symbiosome membrane biogenesis and root nodule development. Further work would identify the additional components of protein trafficking in the early and late secretory pathway in legume roots and nodules and elucidate the mechanism of transport through the Golgi complex to the symbiosome compartment in legumes.

\section{Acknowledgments}

We appreciate the support and laboratory facilities given by Cebi-Tec, Bielefeld University, Bielefeld, Germany. This work was supported by BAP project no. MF010 to A.R. Memon from Usak University, Usak, Turkey.

Clarke, V. C., Loughlin, P. C., Gavrin, A., Chen, C., Brear, E. M., Day, D. A., Smith, P. M. C. 2015: Proteomic analysis of the soybean symbiosome identifies new symbiotic proteins. Molecular and Cellular Proteomics 14, 1301-1322.

Craddock, C., Lavagi, I., Yang, Z. 2012: New insights into Rho signaling from plant ROP/Rac GTPases. Trends in Cell Biology 22, 492-501.

Dalla Via, V., Traubenik, S., Rivero, C., Aguilar, O. M., Zanetti, M. E., Blanco, F. A. 2017: The monomeric GTPase RabA2 is required for progression and maintenance of membrane integrity of infection threads during root nodule symbiosis. Plant Molecular Biology 93, 549-562.

Damiani, I., Drain, A., Guichard, M., Balzergue, S., Boscari, A., Boyer, J.-C., Brunaud, V., Cottaz, S., Rancurel, C., Da Rocha, M., Fizames, C., Fort, S., Gaillard, I., Maillol, V., Danchin, E. G. J., Rouached, H., et al. 2016a: Nod factor effects on root hair-specific transcriptome of medicago truncatula: Focus on plasma membrane transport systems and reactive oxygen species networks. Frontiers in Plant Science 7, 794.

Damiani, I., Pauly, N., Puppo, A., Brouquisse, R., Boscari, A. 2016b: Reactive oxygen species and nitric oxide control early steps of the legume - rhizobium symbiotic interaction. Frontiers in Plant Science 7, 454.

Foreman, J., Demidchik, V., Bothwell, J. H., Mylona, P., Miedema, H., Torres, M. A., Linstead, P., Costa, S., Brownlee, C., Jones, 
J. D., Davies, J. M., Dolan, L. 2003: Reactive oxygen species produced by NADPH oxidase regulate plant cell growth. Nature 422, 442-6.

Fu, Y., Gu, Y., Zheng, Z., Wasteneys, G., Yang, Z. 2005: Arabidopsis interdigitating cell growth requires two antagonistic pathways with opposing action on cell morphogenesis. Cell $120,687-700$.

Gu, Y., Fu, Y., Dowd, P., Li, S., Vernoud, V., Gilroy, S., Yang, Z. 2005: A Rho family GTPase controls actin dynamics and tip growth via two counteracting downstream pathways in pollen tubes. Journal of Cell Biology 169, 127-138.

Ivanov, S., Fedorova, E., Bisseling, T. 2010: Intracellular plant microbe associations: secretory pathways and the formation of perimicrobial compartments. Current Opinion in Plant Biology 13, 372-377.

Jamet, A., Mandon, K., Puppo, A., Herouart, D. 2007: $\mathrm{H}_{2} \mathrm{O}_{2}$ is required for optimal establishment of the Medicago satival Sinorhizobium meliloti symbiosis. Journal of Bacteriology 189, 8741-8745.

Jurgens, G. 2004: Membrane trafficking in plants. Annual Review of Cell Developmental Biology 20, 481- 504.

Ke, D., Li, X., Han, Y., Cheng, L., Yuan, H., Wang, L. 2016: ROP6 is involved in root hair deformation induced by Nod factors in Lotus japonicus. Plant Physiology and Biochemistry 108, 488-498.

Kiirika, L. M., Bergmann, H. F., Schikowsky, C., Wimmer, D., Korte, J., Schmitz, U., Niehaus, K., Colditz, F. 2012: Silencing of the Rac1 GTPase MtROP9 in Medicago truncatula stimulates early mycorrhizal and oomycete root colonizations but negatively affects rhizobial infection. Plant Physiology 159, 501-516.

Lei, M.-J., Wang, Q., Li, X., Chen, A., Luo, L., Xie, Y., Li, G., Luo, D., Mysore, K. S., Wen, J., Xie, Z.-P., Staehelin, C., Wang, Y.Z. 2015: The small GTPase ROP10 of Medicago truncatula is required for both tip growth of root hairs and nod factorinduced root hair deformation. The Plant Cell 27, 806-822.

Limpens, E., Ivanov, S., van Esse, W., Voets, G., Fedorova, E., Bisseling, T. 2009: Medicago $\mathrm{N}_{2}$-fixing symbiosomes acquire the endocytic identity marker Rab7 but delay the acquisition of vacuolar identity. The Plant Cell 21, 2811-2828.

Liu, W., Chen, A. M., Luo, L., Sun, J., Cao, L. P., Yu, G. Q., Zhu, J. B., Wang, Y. Z. 2010: Characterization and expression analysis of Medicago truncatula ROP GTPase family during the early stage of symbiosis. Journal of Integrative Plant Biology $52,639-52$.

Lycett, G. 2008: The role of Rab GTPases in cell wall metabolism. Journal of Experimental Botany 59, 4061-4074.

Marino, D., Andrio, E., Danchin, E. G. J., Oger, E., Gucciardo, S., Lambert, A., Puppo, A. Pauly, N. 2011: A Medicago truncatula NADPH oxidase is involved in symbiotic nodule functioning. New Phytologist 189, 580-592.

Matheson, L. A., Hanton, S. L., Rossi, M., Latijnhouwers, M., Stefano, G., Renna, L., Brandizzi, F. 2007: Multiple roles of ADPribosylation factor 1 in plant cells include spatially regulated recruitment of coatomer and elements of the Golgi matrix. Plant Physiology 143, 1615-1627.

Mazel, A., Leshem, Y., Tiwari, B. S., Levine, A. 2004: Induction of salt and osmotic stress tolerance by overexpression of an intracellular vesicle trafficking protein AtRab7 (AtRabG3e). Plant Physiology 134, 118-128.

Mehrtens, F., Kranz, H., Bednarek, P., Weisshaar, B. 2005: The Arabidopsis transcription factor MYB12 is a flavonol-specific regulator of phenylpropanoid biosynthesis. Plant Physiology $138,1083-1096$.

Memon, A. R. 2004: The role of ADP-ribosylation factor and SAR1 in vesicular trafficking in plants. Biochimica et Biophysica Acta 1664, 9-30.
Memon, A. R. 2012: Transcriptomics and proteomics analysis of root nodules of model legume plants. In: Ashraf, M., Ozturl, M., Ahmed, S. M. A., Aksoy, A (eds.), Crop production for agricultural improvement, Springer, 291-315.

Montiel, J., Nava, N., Cárdenas, L., Sánchez-López, R., Arthikala, M. K., Santana, O., Sánchez, F., Quinto, C. 2012: A Phaseolus vulgaris NADPH oxidase gene is required for root infection by rhizobia. Plant and Cell Physiology 53, 1751-1767.

Navazio, L., Moscatiello, R., Genre, A., Novero, M., Baldan, B., Bonfante, P., Mariani, P. 2007: A diffusible signal from arbuscular mycorrhizal fungi elicits a transient cytosolic calcium elevation in host plant cells. Plant Physiology 144, 673-681.

Oldroyd, G. E., Downie, J. A. 2008: Coordinating nodule morphogenesis with rhizobial infection in legumes. Annual Review of Plant Biology 59, 519-546.

Park, M., Jürgens, G. 2012: Membrane traffic and fusion at postGolgi compartments. Frontiers in Plant Science 2, 111.

Pauly, N., Pucciariello, C., Mandon, K., Innocenti, G., Jamet, A., Baudouin, E., Herouart, D., Frendo, P., Puppo, A. 2006: Reactive oxygen and nitrogen species and glutathione: key players in the legume-Rhizobium symbiosis. Journal of Experimental Botany 57, 1769-1776.

Pfeffer, S. R. 2017: Rab GTPases: master regulators that establish the secretory and endocytic pathways. Molecular Biology of the Cell 28, 712-715.

Pimpl, P., Movafeghi, A., Coughlan, S., Denecke, J., Hillmer, S., Robinson, D. G. 2000: In situ localization and in vitro induction of plant COPI-coated vesicles. Plant Cell 12, 2219-2236.

Potocky, M., Jones, M. A., Bezvoda, R., Smirnoff, N., Zarsky, V. 2007: Reactive oxygen species produced by NADPH oxidase are involved in pollen tube growth. New Phytologist $174,742-751$.

Riely, B. K., He, H., Venkateshwaran, M., Sarma, B., Schraiber, J., Ane, J. M., Cook, D. R. 2011: Identification of legume RopGEF gene families and characterization of a Medicago truncatula Rop GEF mediating polar growth of root hairs. Plant Journal 65, 230-243.

Rolfe, B. G., Gresshoff, P. M., Shine, J. 1980: Rapid screening for symbiotic mutants of Rhizobium and white clover. Plant Science Letters 19, 277-284.

Rozen, S., Skaletsky, H. 2000: Primer3 on the WWW for general users and for biologist programmers. Methods in Molecular Biology 132, 365-386.

Schiene, K., Donath, S., Brecht, M., Puhler, A., Niehaus, K. 2004: A Rab-related small GTP binding protein is predominantly expressed in root nodules of Medicago sativa. Molecular Genetics and Genomics 272, 57- 66.

Son, O., Yang, H.-S., Lee, H.-J., Lee, M.-Y., Shin, K.-H., Jeon, S.-L., Lee, M.-S., Choi, S.-Y., Chun, J.-Y., Kim, H., An, C.-S., Hong, S.-K., Kim, N.-S., Koh, S.-K., Cho, M. J., Kim, S., et al. 2003: Expression of srab7 and SCaM genes required for endocytosis of Rhizobium in root nodules. Plant Science 165, 1239-1244.

Szumlanski, A. L., Nielsen, E. 2009: The Rab GTPase RabA4d regulates pollen tube tip growth in Arabidopsis thaliana. Plant Cell 21, 526-544.

Tesfaye, M., Samac, D. A., Vance, C. P. 2006: Insights into symbiotic nitrogen fixation in Medicago truncatula. Molecular Plant Microbe Interaction 19, 330-341.

Tichopad, A., Dilger, M., Schwarz, G., Pfaffl, M. W. 2003: Standardized determination of real-time PCR efficiency from a single reaction set-up. Nucleic Acids Research 31, e122.

Vernoud, V., Horton, A. C., Yang, Z., Nielsen, E. 2003: Analysis of the small GTPase gene superfamily of Arabidopsis. Plant Physiology 131, 1191-1208.

Wang, D., Griffitts, J., Starker, C., Fedorova, E., Limpens, E., Ivanov, S., Bisseling, T., Long, S. 2010: A nodule-specific protein 
secretory pathway required for nitrogen-fixing symbiosis. Science 327, 1126-1129.

Wong, H. L., Pinontoan, R., Hayashi, K., Tabata, R., Yaeno, T., Hasegawa, K., Kojima, C., Yoshioka, H., Iba, K., Kawasaki, T., Shimamoto, K. 2007: Regulation of rice NADPH oxidase by binding of Rac GTPase to its N-terminal extension. Plant Cell 19, 4022-4034.

Woollard, A. A., Moore, I. 2008: The functions of Rab GTPases in plant membrane traffic. Current Opinion in Plant Biology $11,610-619$.
Yalovsky, S., Bloch, D., Sorek, N., Kost, B. 2008: Regulation of membrane trafficking, cytoskeleton dynamics, and cell polarity by ROP/RAC GTPases. Plant Physiology 147, 15271543.

Yuksel, B., Memon, A. R. 2008: Comparative phylogenetic analysis of small GTP-binding genes of model legume plants and assessment of their roles in root nodules. Journal of Experimental Botany 59, 3831-3844. 